electricians of 0.71 (95\%CI $0.48-1.06)$. Total person months at risk (excluding months with no sex, pregnancy, surgical sterility or menopause) were 12696 for welders and 13313 for electricians. Likelihood of conceiving in any month at risk was significantly reduced with use of better contraception, drinking $>7$ units of alcohol, increasing age, number of previous pregnancies and, for welders, smoking >10 cigarettes/day. No effect of either current or cumulative work in trade was evident having adjusted for confounding. HR for working as a welder was -0.07 95\% CI $-0.49-0.35$.

Conclusions Female infertility presents challenges as an outcome in occupational epidemiology, but data can be structured to allow for time at risk. Analysis to date has demonstrated no important effect of exposure to welding.

\section{B.2 PARENTAL OCCUPATIONAL EXPOSURE TO EXTREMELY LOW-FREQUENCY MAGNETIC FIELDS AND THE RISK OF LEUKAEMIA IN THE OFFSPRING}

Madar Talibov. International Agency for Research on Cancer, Lyon, France

\subsection{6/OEM-2019-EPI.13}

Background Leukaemia is the most common cancer diagnosed in children worldwide, accounting for about one third of all paediatric malignancies in economically developed countries. Despite extensive research, the aetiology of this cancer remains largely unknown. Previous studies suggested that parental occupational exposure to extremely low-frequency magnetic fields (ELF-MF) may increase leukaemia risk in the offspring.

Objective We aimed to evaluate the association of parental occupational exposure to ELF-MF with the risk of acute lymphoblastic leukaemia (ALL) and acute myeloid leukaemia (AML) in their offspring.

Methods We pooled data from 11 case-control studies participating in the Childhood Leukaemia International Consortium (CLIC). The studies included 9723 childhood leukaemia cases and 17099 controls. Data on parental job history and other characteristics of the study population were collected by questionnaires and interviews, except for the Finnish study where data was obtained from population registries and censuses. Parental occupational ELF-MF exposure was estimated by linking job histories to an independently developed international ELF-MF job-exposure matrix. Logistic regression models were used to estimate odds ratios (OR) and 95\% confidence intervals $(95 \% \mathrm{CI})$. We employed two analytical approaches to obtain summary risk estimates. First, we conducted pooled analyses of all studies combined. Second, we summarized ORs from individual studies in meta-analyses.

Results The summary OR estimates from pooled analyses for paternal ELF-MF exposure at conception were 1.04 (95\% CI 0.95-1.13) for ALL and 1.06 (95\% CI 0.87-1.29) for AML, at $>0.2$ microtesla $(\mu \mathrm{T})$ compared to $\leq 0.2 \mu \mathrm{T}$. Corresponding estimates for maternal ELF-MF exposure during pregnancy, using again the $0.2 \mu \mathrm{T}$ cut-off, were 1.00 (95\% CI $0.89-1.12$ ) for ALL and 0.85 (95\% CI 0.61-1.16) for AML. No trends with increasing exposure level were evident. Furthermore, no associations were observed in the meta-analyses.

Conclusion Our study did not find any associations between parental occupational ELF-MF exposure and childhood leukaemia.
01B.3 HYPERACTIVITY DISORDER IN CHILDREN WAS RELATED TO MATERNAL EMPLOYMENT STATUS DURING PREGNANCY AND POSTPARTUM DEPRESSIVE SYMPTOMS: A PROSPECTIVE COHORT STUDY

${ }^{1,2}$ Ping Shih*, ${ }^{1,2}$ Ching-Chun Huang, ${ }^{3}$ Tung-liang Chiang, ${ }^{1,2}$ Pau-Chung Chen, ${ }^{1,2,4}$ YueLiang Leon Guo. 'Department of Environmental and Occupational Medicine, National Taiwan University (NTU) College of Medicine and NTU Hospital, Taipei, Taiwan; ${ }^{2}$ Institute of Occupational Medicine and Industrial Hygiene, College of Public Health, National Taiwan University, Taipei, Taiwan; ${ }^{3}$ Institute of Health Policy and Management, College of Public Health, National Taiwan University, Taipei, Taiwan; ${ }^{4}$ National Institute of Environmental Health Sciences, National Health Research Institutes, Zhunan, Taiwan

\subsection{6/OEM-2019-EPI.14}

Background Attention-deficit/hyperactivity disorder (ADHD) is one of the most common neurobehavioral disorders globally. Although some investigations implied a relationship between $\mathrm{ADHD}$ and maternal psychosocial stress exposure during pregnancy, little is known about the effects of maternal occupational exposure and even postpartum mental health. This study aimed to investigate whether maternal employment status during pregnancy and postpartum depressive symptoms are related to offspring hyperactivity, one of the key early symptoms of children ADHD.

Methods Taiwan Birth Cohort Study recruited representative mother-infant pairs, as a result of approximately $12 \%$ of all deliveries in 2005 using multistage stratified sampling. Employment status with or without job stress during pregnancy and postpartum depressive symptoms were inquired when the child was six months of age by face-to-face interview. Ever having hyperactivity syndrome as evaluated by physicians, psychologists, or special educators was inquired when the child was eight years of age. Factors of hyperactivity, including maternal employment, job stress, and postpartum depression were studied by adjusted odds ratios (aORs) and 95\% confidence interval (CI) using logistic regression, adjusting for gender, urban/rural residence, birth season and household income.

Results 18215 mother-infant pairs were included in the final analysis, where 421 (2.3\%) of children had been diagnosed as having hyperactivity before 8 years of age. Comparing to mothers employed and without job stress during pregnancy, the aOR (95\% CI) of child hyperactivity was 1.47 (95\%CI: $1.12,1.94)$ for mothers with job stress and 1.43 (95\%CI: $1.12,1.84)$ for mothers with no employment during pregnancy, respectively. Besides, children were 1.36 (95\% CI: $1.07,1.73)$ times more likely to receive an ADHD diagnosis if their mother experienced postpartum depressive symptoms.

Conclusions In this prospective birth cohort study, mothers' employment status, job stress during pregnancy, and postpartum depressive symptoms were risk factors for the occurrence of hyperactivity in their children.

\section{B.4 HEALTH EFFECTS OF PRENATAL OCCUPATIONAL NOISE EXPOSURE: A SYSTEMATIC REVIEW}

Zara Ann Stokholm*, Inge Brosbøl Iversen, Henrik Kolstad. Department of Occupational Medicine, Aarhus University Hospital, Aarhus N, Denmark

\subsection{6/OEM-2019-EPI.15}

Current legislation and threshold limits for occupational noise exposure may not sufficiently account for higher vulnerability of the foetus. We conducted a systematic literature review and 